\title{
HOMOTOPY ON SPATIAL GRAPHS AND GENERALIZED SATO-LEVINE INVARIANTS
}

\author{
RYO NIKKUNI
}

\begin{abstract}
Edge-homotopy and vertex-homotopy are equivalence relations on spatial graphs which are generalizations of Milnor's link-homotopy. Fleming and the author introduced some edge (resp. vertex)-homotopy invariants of spatial graphs by applying the Sato-Levine invariant for the constituent 2component algebraically split links. In this paper, we construct some new edge (resp. vertex)-homotopy invariants of spatial graphs without any restriction of linking numbers of the constituent 2-component links by applying the generalized Sato-Levine invariant.
\end{abstract}

\section{INTRODUCTION}

Throughout this paper we work in the piecewise linear category. Let $G$ be a finite graph. An embedding $f$ of $G$ into the 3 -sphere $\mathbb{S}^{3}$ is called a spatial embedding of $G$ or simply a spatial graph. We call the image of $f$ restricted on a cycle (resp. mutually disjoint cycles) in $G$ a constituent knot (resp. constituent link) of $f$, where a cycle is a graph homeomorphic to a circle. A spatial embedding of a planar graph is said to be trivial if it is ambient isotopic to an embedding of the graph into a 2-sphere in $\mathbb{S}^{3}$. A spatial embedding $f$ of $G$ is said to be split if there exists a 2-sphere $S$ in $\mathbb{S}^{3}$ such that $S \cap f(G)=\emptyset$ and each connected component of $\mathbb{S}^{3}-S$ has intersection with $f(G)$, and otherwise $f$ is said to be non-splittable.

Two spatial embeddings of $G$ are said to be edge-homotopic if they are transformed into each other by self crossing changes and ambient isotopies, where a self crossing change is a crossing change on the same spatial edge, and vertex-homotopic if they are transformed into each other by crossing changes on two adjacent spatial edges and ambient isotopies. These equivalence relations were introduced by Taniyama [17] as generalizations of Milnor's link-homotopy on oriented links [12, namely if $G$ is a mutually disjoint union of cycles then these are none other than link-homotopy. It is known that edge (resp. vertex)-homotopy on spatial graphs behaves quite differently than link-homotopy on oriented links. Taniyama introduced the $\alpha$-invariant of spatial graphs by taking a weighted sum of the second coefficient of the Conway polynomial of the constituent knots [16]. By applying the $\alpha$-invariant, it is shown that the spatial embedding of $K_{4}$ as illustrated in Fig. 1.1 (1) is not trivial up to edge-homotopy, and two spatial embeddings of $K_{3,3}$ as illustrated in Fig. 1.1 (2) and (3) are not vertex-homotopic. Note that each of these spatial graphs does not have a consituent link. On the other hand, some invariants of spatial graphs defined by taking a weighted sum of the third coefficient of the Conway polynomial of the constituent 2-component links were introduced

1991 Mathematics Subject Classification. Primary 57M15; Secondary 57M25.

Key words and phrases. Spatial graph, edge-homotopy, vertex-homotopy, generalized SatoLevine invariant. 
by Taniyama as $\mathbb{Z}_{2}$-valued invariants if the linking numbers are even [18, and by Fleming and the author as integer-valued invariants if the linking numbers vanish 4. By applying these invariants, it is shown that each of the spatial graphs as illustrated in Fig. 1.2 (1) and (2) is non-splittable up to edge-homotopy, and the spatial graph as illustrated in Fig. 1.2 (3) is non-splittable up to vertex-homotopy. Note that each of these spatial graphs does not contain a constituent link which is not trivial up to link-homotopy.

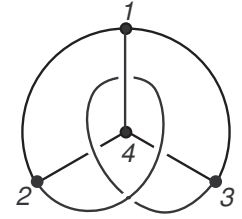

(1)

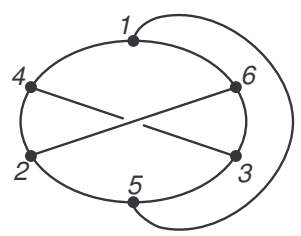

(2)

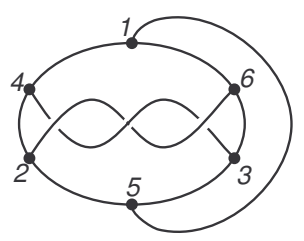

(3)

Figure 1.1

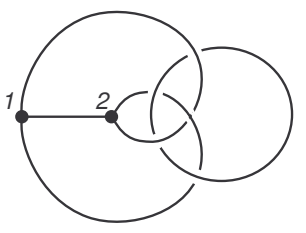

(1)



(2)

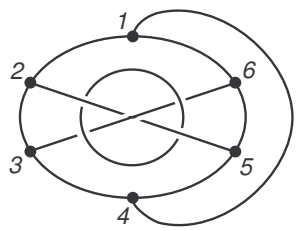

(3)

FiguRE 1.2.

Our purpose in this paper is to construct some new edge (resp. vertex)-homotopy invariants of spatial graphs without any restriction of linking numbers of the constituent 2-component links by applying a weighted sum of the generalized SatoLevine invariant. Here the generalized Sato-Levine invariant $\tilde{\beta}(L)=\tilde{\beta}\left(K_{1}, K_{2}\right)$ is an ambient isotopy invariant of an oriented 2-component link $L=K_{1} \cup K_{2}$ which appears in various ways independently [1], 2], 10], 11, [9, [13] and can be calculated by

$$
\tilde{\beta}(L)=a_{3}(L)-\operatorname{lk}(L)\left\{a_{2}\left(K_{1}\right)+a_{2}\left(K_{2}\right)\right\},
$$

where $a_{i}$ denotes the $i$-th coefficient of the Conway polynomial and $\operatorname{lk}(L)=\operatorname{lk}\left(K_{1}, K_{2}\right)$ denotes the linking number of $L$. It is known that the original Sato-Levine invariant $\beta(L)$ [14] coincides with $a_{3}(L)$ if $\operatorname{lk}(L)=0$ [3, 15]. Thus in this case we have that $\tilde{\beta}(L)=\beta(L)$. As a consequence, our invariants are generalizations of Fleming and the author's homotopy invariants of spatial graphs defined in [4.

This paper is organized as follows. In the next section, we show some formulas about the generalized Sato-Levine invariant of oriented 2-component links needed later. In section 3, we give the definitions of our invariants and state their invariance up to edge (resp. vertex)-homotopy. In section 4, we give some examples. 


\section{Some formulas about the Generalized Sato-Levine invariant}

We first show the change in the generalized Sato-Levine invariant of oriented 2-component links which differ by a single self crossing change.

Lemma 2.1. Let $L_{+}=J_{+} \cup K$ and $L_{-}=J_{-} \cup K$ be two oriented 2 -component links and $L_{0}=J_{1} \cup J_{2} \cup K$ an oriented 3 -component link which are identical except inside the depicted regions as illustrated in Fig. 2.1. Suppose that $\operatorname{lk}\left(L_{+}\right)=\operatorname{lk}\left(L_{-}\right)=m$. Then it holds that

$$
\tilde{\beta}\left(L_{+}\right)-\tilde{\beta}\left(L_{-}\right)=\operatorname{lk}\left(K, J_{i}\right)\left\{m-\operatorname{lk}\left(K, J_{i}\right)\right\} \quad(i=1,2) .
$$

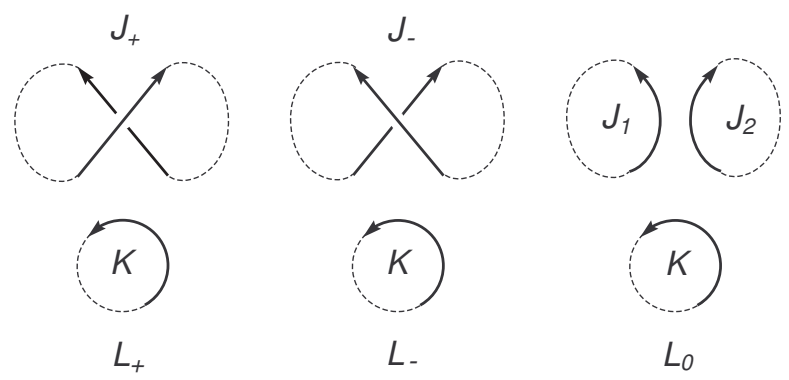

FIGURE 2.1.

We remark here that this formula has already known (see [11, Theorem 8.7] for example), but we give the proof again for readers' convenience.

Proof. By the skein relation of the Conway polynomial and well-known formulas for the first coefficient of the Conway polynomial of an oriented 2-component link (cf. [8]) and for the second coefficient of the Conway polynomial of an oriented 3-component link (cf. [6], [5], 7]), we have that

$$
\begin{aligned}
a_{2}\left(J_{+}\right)-a_{2}\left(J_{-}\right)= & \operatorname{lk}\left(J_{1}, J_{2}\right), \\
a_{3}\left(L_{+}\right)-a_{3}\left(L_{-}\right)= & \operatorname{lk}\left(J_{1}, J_{2}\right) \operatorname{lk}\left(J_{2}, K\right)+\operatorname{lk}\left(J_{2}, K\right) \operatorname{lk}\left(J_{1}, K\right) \\
& +\operatorname{lk}\left(J_{1}, K\right) \operatorname{lk}\left(J_{1}, J_{2}\right) .
\end{aligned}
$$

Note that

$$
\operatorname{lk}\left(J_{1}, K\right)+\operatorname{lk}\left(J_{2}, K\right)=m .
$$

Thus by (2.1), (2.2) and (2.3), we have that

$$
\begin{aligned}
\tilde{\beta}\left(L_{+}\right)-\tilde{\beta}\left(L_{-}\right)= & a_{3}\left(L_{+}\right)-m\left\{a_{2}\left(J_{+}\right)+a_{2}(K)\right\}-a_{3}\left(L_{-}\right)+m\left\{a_{2}\left(J_{-}\right)+a_{2}(K)\right\} \\
= & a_{3}\left(L_{+}\right)-a_{3}\left(L_{-}\right)-m\left\{a_{2}\left(J_{+}\right)-a_{2}\left(J_{-}\right)\right\} \\
= & \operatorname{lk}\left(J_{1}, J_{2}\right)\left\{m-\operatorname{lk}\left(J_{1}, K\right)\right\}+\operatorname{lk}\left(J_{2}, K\right) \operatorname{lk}\left(J_{1}, K\right) \\
& +\operatorname{lk}\left(J_{1}, K\right) \operatorname{lk}\left(J_{1}, J_{2}\right)-m \operatorname{lk}\left(J_{1}, J_{2}\right) \\
= & \operatorname{lk}\left(J_{2}, K\right) \operatorname{lk}\left(J_{1}, K\right) .
\end{aligned}
$$

Therefore by (2.3) we have the result. 
Next we investigate the change in the generalized Sato-Levine invariant of oriented 2-component links which differ by inverting the orientation on one of the components. The original definition implies that the value of the Sato-Levine invariant does not depend on the orientation of each component. But the value of the generalized Sato-Levine invariant depends on it in general.

Theorem 2.2. Let $L=J_{1} \cup J_{2}$ be an oriented 2-component link with $\operatorname{lk}(L)=m$. Let $L^{\prime}=\left(-J_{1}\right) \cup J_{2}$ be the oriented 2 -component link obtained from $L$ by inverting the orientation of $J_{1}$. Then it holds that

$$
\tilde{\beta}(L)-\tilde{\beta}\left(L^{\prime}\right)=\frac{1}{6}\left(m^{3}-m\right) .
$$

Proof. Let $T_{m}$ and $T_{m}^{\prime}$ be two oriented 2-component links as illustrated in Fig. 2.2 . By a direct calculation we have that $\operatorname{lk}\left(T_{m}\right)=m, \operatorname{lk}\left(T_{m}^{\prime}\right)=-m$ and

$$
\begin{aligned}
& a_{3}\left(T_{m}\right)=\frac{1}{6}\left(m^{3}-m\right), \\
& a_{3}\left(T_{m}^{\prime}\right)=0 .
\end{aligned}
$$

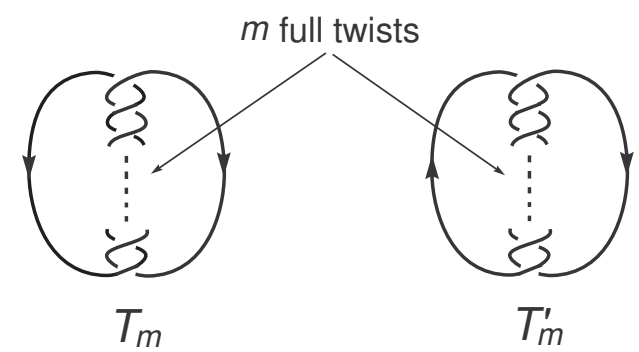

FIGURE 2.2.

By the classification of oriented 2-component links up to link-homotopy [12, we have that $L$ and $T_{m}$ are transformed into each other by self crossing changes and ambient isotopies. Namely there exists a sequence of oriented 2-component links $L=L_{0}, L_{1}, \ldots, L_{k-1}, L_{k}=T_{m}$ such that $L_{i}$ is obtained from $L_{i-1}$ by a single self crossing change $c_{i}(i=1,2, \ldots, k)$. Let $M_{i}=K_{0}^{(i)} \cup K_{1}^{(i)} \cup K_{2}^{(i)}$ be the oriented 3 -component link obtained from $L_{i-1}$ by smoothing it on $c_{i}$, where $K_{1}^{(i)}$ and $K_{2}^{(i)}$ are actual separated parts $(i=1,2, \ldots, k)$. Namely we have the upper part of a skein tree as follows.

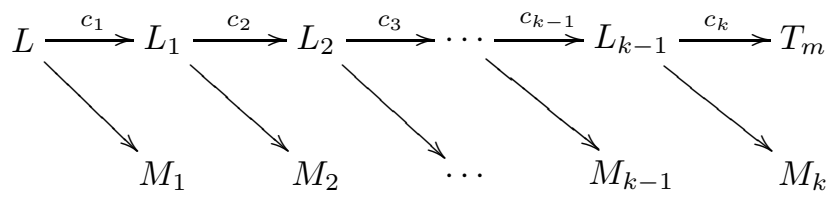

We define a sign of $c_{i}$ as follows: $\varepsilon\left(c_{i}\right)=1$ if $c_{i}$ changes a positive crossing into a negative crossing and -1 if $c_{i}$ changes a negative crossing into a positive crossing. 
HOMOTOPY ON SPATIAL GRAPHS AND GENERALIZED SATO-LEVINE INVARIANTS 5

Since $\operatorname{lk}\left(L_{i}\right)=m(i=1, \ldots, k)$, we have that

$$
\operatorname{lk}\left(K_{0}^{(i)}, K_{1}^{(i)}\right)+\operatorname{lk}\left(K_{0}^{(i)}, K_{2}^{(i)}\right)=m
$$

for $i=1,2, \ldots, k$. Then by (2.4), (2.6) and (2.7), we have that

$$
\begin{aligned}
a_{3}(L)= & a_{3}\left(T_{m}\right)+\sum_{i=1}^{k} \varepsilon\left(c_{i}\right) a_{2}\left(M_{i}\right) \\
= & \frac{1}{6}\left(m^{3}-m\right)+\sum_{i=1}^{k} \varepsilon\left(c_{i}\right)\left\{\operatorname{lk}\left(K_{0}^{(i)}, K_{1}^{(i)}\right) \operatorname{lk}\left(K_{1}^{(i)}, K_{2}^{(i)}\right)\right. \\
& \left.+\operatorname{lk}\left(K_{1}^{(i)}, K_{2}^{(i)}\right) \operatorname{lk}\left(K_{0}^{(i)}, K_{2}^{(i)}\right)+\operatorname{lk}\left(K_{0}^{(i)}, K_{2}^{(i)}\right) \operatorname{lk}\left(K_{0}^{(i)}, K_{1}^{(i)}\right)\right\} \\
= & \frac{1}{6}\left(m^{3}-m\right)+\sum_{i=1}^{k} \varepsilon\left(c_{i}\right)\left(\left\{m-\operatorname{lk}\left(K_{0}^{(i)}, K_{2}^{(i)}\right)\right\} \operatorname{lk}\left(K_{1}^{(i)}, K_{2}^{(i)}\right)\right. \\
& \left.+\operatorname{lk}\left(K_{1}^{(i)}, K_{2}^{(i)}\right) \operatorname{lk}\left(K_{0}^{(i)}, K_{2}^{(i)}\right)+\operatorname{lk}\left(K_{0}^{(i)}, K_{2}^{(i)}\right) \operatorname{lk}\left(K_{0}^{(i)}, K_{1}^{(i)}\right)\right) \\
& \frac{1}{6}\left(m^{3}-m\right)+\sum_{i=1}^{k} \varepsilon\left(c_{i}\right)\left\{m \operatorname{lk}\left(K_{1}^{(i)}, K_{2}^{(i)}\right)+\operatorname{lk}\left(K_{0}^{(i)}, K_{2}^{(i)}\right) \operatorname{lk}\left(K_{0}^{(i)}, K_{1}^{(i)}\right)\right\} .
\end{aligned}
$$

In the same way as above, we have that $L^{\prime}$ and $T_{m}^{\prime}$ are transformed into each other by self crossing changes and ambient isotopies and obtain the following skein tree from (2.6) immediately:

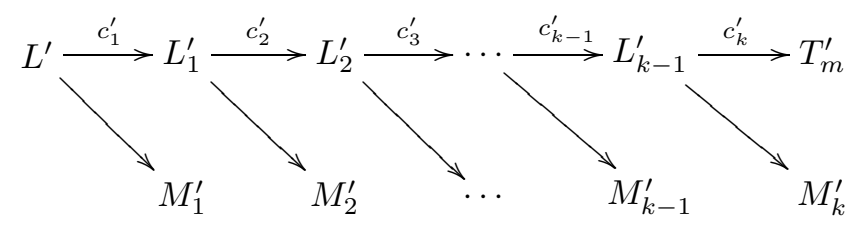

We also denote $M_{i}^{\prime}={K^{\prime}}_{0}^{(i)} \cup K^{\prime(i)} \cup K^{\prime(i)}$, where $K_{1}^{\prime(i)}$ and ${K^{\prime}}_{2}^{(i)}$ are actual separated parts obtained by smoothing $L_{i-1}^{\prime}$ on $c_{i}^{\prime}(i=1,2, \ldots, k)$. Note that

$$
\begin{aligned}
\varepsilon\left(c_{i}\right) & =\varepsilon\left(c_{i}^{\prime}\right), \\
\operatorname{lk}\left(K_{1}^{(i)}, K_{2}^{(i)}\right) & =\operatorname{lk}\left({K^{\prime}}_{1}^{(i)}, K_{2}^{\prime(i)}\right) \\
\operatorname{lk}\left(K_{0}^{(i)}, K_{1}^{(i)}\right) \operatorname{lk}\left(K_{0}^{(i)}, K_{2}^{(i)}\right) & =\operatorname{lk}\left({K^{\prime}}_{0}^{(i)},{K^{\prime}}_{1}^{(i)}\right) \operatorname{lk}\left({K^{\prime}}_{0}^{(i)}, K_{2}^{\prime(i)}\right)
\end{aligned}
$$

for $i=1,2, \ldots, k$. Then by (2.5), (2.9), (2.10), (2.11) and (2.12), we have that

$$
\begin{aligned}
a_{3}\left(L^{\prime}\right) & =a_{3}\left(T_{m}^{\prime}\right)+\sum_{i=1}^{k} \varepsilon\left(c_{i}^{\prime}\right) a_{2}\left(M_{i}^{\prime}\right) \\
& =\sum_{i=1}^{k} \varepsilon\left(c_{i}^{\prime}\right)\left\{-m \operatorname{lk}\left({K^{\prime}}_{1}^{(i)}, K_{2}^{\prime(i)}\right)+\operatorname{lk}\left({K_{0}^{\prime}}_{0}^{(i)}, K_{2}^{\prime(i)}\right) \operatorname{lk}\left(K_{0}^{\prime(i)}, K_{1}^{\prime(i)}\right)\right\} \\
& =\sum_{i=1}^{k} \varepsilon\left(c_{i}\right)\left\{-m \operatorname{lk}\left(K_{1}^{(i)}, K_{2}^{(i)}\right)+\operatorname{lk}\left(K_{0}^{(i)}, K_{2}^{(i)}\right) \operatorname{lk}\left(K_{0}^{(i)}, K_{1}^{(i)}\right)\right\} .
\end{aligned}
$$


On the other hand, by (2.6) we can see easily that

$$
a_{2}\left(J_{1}\right)+a_{2}\left(J_{2}\right)=\sum_{i=1}^{k} \varepsilon\left(c_{i}\right) \operatorname{lk}\left(K_{1}^{(i)}, K_{2}^{(i)}\right) .
$$

By combining (2.8), (2.13) and (2.14), we have that

$$
\begin{aligned}
a_{3}(L)-a_{3}\left(L^{\prime}\right) & =\frac{1}{6}\left(m^{3}-m\right)+2 m \sum_{i=1}^{k} \varepsilon\left(c_{i}\right) \operatorname{lk}\left(K_{1}^{(i)}, K_{2}^{(i)}\right) \\
& =\frac{1}{6}\left(m^{3}-m\right)+2 m\left\{a_{2}\left(J_{1}\right)+a_{2}\left(J_{2}\right)\right\} .
\end{aligned}
$$

Then by (2.15), we have that

$$
\begin{aligned}
\tilde{\beta}(L)-\tilde{\beta}\left(L^{\prime}\right) & =a_{3}(L)-m\left\{a_{2}\left(J_{1}\right)+a_{2}\left(J_{2}\right)\right\}-a_{3}\left(L^{\prime}\right)-m\left\{a_{2}\left(-J_{1}\right)+a_{2}\left(J_{2}\right)\right\} \\
& =a_{3}(L)-a_{3}\left(L^{\prime}\right)-2 m\left\{a_{2}\left(J_{1}\right)+a_{2}\left(J_{2}\right)\right\} \\
& =\frac{1}{6}\left(m^{3}-m\right) .
\end{aligned}
$$

This completes the proof.

Remark 2.3. Let $f$ be a spatial embedding of a graph $G$ and $\gamma, \gamma^{\prime}$ two disjoint cycles of $G$. By Theorem 2.2, if $\operatorname{lk}\left(f(\gamma), f\left(\gamma^{\prime}\right)\right)=0, \pm 1$ then the value of $\tilde{\beta}\left(f(\gamma), f\left(\gamma^{\prime}\right)\right)$ does not depend on the orientation of $f(\gamma)$ and $f\left(\gamma^{\prime}\right)$, namely it is well-defined. But if $\operatorname{lk}\left(f(\gamma), f\left(\gamma^{\prime}\right)\right) \neq 0$, then Theorem 2.2 implies that the value of $\tilde{\beta}\left(f(\gamma), f\left(\gamma^{\prime}\right)\right)$ have the indeterminacy arisen from a choice of the orientations of $f(\gamma)$ and $f\left(\gamma^{\prime}\right)$.

\section{Definitions of invariants}

From now onward, we assume that a graph $G$ is oriented, namely an orientation is given for each edge of $G$. For a subgraph $H$ of $G$, we denote the set of all cycles of $H$ by $\Gamma(H)$. For an edge $e$ of $H$, we denote the set of all oriented cycles of $H$ which contain the edge $e$ and have the orientation induced by the orientation of $e$ by $\Gamma_{e}(H)$. For a pair of two adjacent edges $e$ and $e^{\prime}$ of $H$, we denote the set of all oriented cycles of $H$ which contain the edges $e$ and $e^{\prime}$ and have the orientation induced by the orientation of $e$ by $\Gamma_{e, e^{\prime}}(H)$. We set $\mathbb{Z}_{n}=\{0,1, \ldots, n-1\}$ for a positive integer $n$ and $\mathbb{Z}_{0}=\mathbb{Z}$. We call a map $\omega: \Gamma(H) \rightarrow \mathbb{Z}_{n}$ a weight on $\Gamma(H)$ over $\mathbb{Z}_{n}$. Then we say that a weight $\omega$ on $\Gamma(H)$ over $\mathbb{Z}_{n}$ is weakly balanced on an edge $e$ if

$$
\sum_{\gamma \in \Gamma_{e}(H)} \omega(\gamma) \equiv 0 \quad(\bmod n),
$$

and weakly balanced on a pair of adjacent edges $e$ and $e^{\prime}$ if

$$
\sum_{\gamma \in \Gamma_{e, e^{\prime}}(H)} \omega(\gamma) \equiv 0 \quad(\bmod n) .
$$

Let $G=G_{1} \cup G_{2}$ be a disjoint union of two graphs, $\omega_{i}$ a weight on $\Gamma\left(G_{i}\right)$ over $\mathbb{Z}_{n}(i=1,2)$ and $f$ a spatial embedding of $G$. Then we say that a weight $\omega_{i}$ is 
null-homologous on an edge e of $G_{i}$ with respect to $f$ and $\omega_{j}(i \neq j)$ if

$$
\mathrm{lk}\left(\sum_{\gamma \in \Gamma_{e}\left(G_{i}\right)} \omega_{i}(\gamma) f(\gamma), f\left(\gamma^{\prime}\right)\right) \equiv 0 \quad(\bmod n)
$$

for any $\gamma^{\prime} \in \Gamma\left(G_{j}\right)$ with $\omega_{j}\left(\gamma^{\prime}\right) \neq 0$, and null-homologous on a pair of adjacent edges $e$ and $e^{\prime}$ of $G_{i}$ with respect to $f$ and $\omega_{j}(i \neq j)$ if

$$
\operatorname{lk}\left(\sum_{\gamma \in \Gamma_{e, e^{\prime}}\left(G_{i}\right)} \omega_{i}(\gamma) f(\gamma), f\left(\gamma^{\prime}\right)\right) \equiv 0 \quad(\bmod n)
$$

for any $\gamma^{\prime} \in \Gamma\left(G_{j}\right)$ with $\omega_{j}\left(\gamma^{\prime}\right) \neq 0$.

Example 3.1. Let $G=G_{1} \cup G_{2}$ be the graph as illustrated in Fig. 3.1. We denote the cycle $e_{i} \cup e_{j}$ of $G_{1}$ by $\gamma_{i j}$. Let $\omega_{1}$ be the weight on $\Gamma\left(G_{1}\right)$ over $\mathbb{Z}$ defined by

$$
\omega_{1}(\gamma)= \begin{cases}1 & \left(\gamma=\gamma_{12}, \gamma_{34}\right) \\ -1 & \left(\gamma=\gamma_{23}, \gamma_{14}\right) \\ 0 & \text { (otherwise) }\end{cases}
$$

and $\omega_{2}$ the weight on $\Gamma\left(G_{2}\right)$ over $\mathbb{Z}$ defined by $\omega_{2}\left(\gamma^{\prime}\right)=1$. Let $f$ be the spatial embedding of $G$ as illustrated in Fig. 3.1. Note that

$$
\Gamma_{e_{1}}\left(G_{1}\right)=\left\{\gamma_{12}, \gamma_{13}, \gamma_{14}\right\}=\left\{e_{1}+e_{2}, e_{1}-e_{3}, e_{1}+e_{4}\right\}
$$

and

$$
\sum_{\gamma \in \Gamma_{e_{1}}\left(G_{1}\right)} \omega_{1}(\gamma) \gamma=\left(e_{1}+e_{2}\right)-\left(e_{1}+e_{4}\right)=e_{2}-e_{4}
$$

Then we have that

$$
\operatorname{lk}\left(\sum_{\gamma \in \Gamma_{e_{1}}\left(G_{1}\right)} \omega_{1}(\gamma) f(\gamma), f\left(\gamma^{\prime}\right)\right)=\operatorname{lk}\left(f\left(e_{2}-e_{4}\right), f\left(\gamma^{\prime}\right)\right)=0 .
$$

Therefore $\omega_{1}$ is null-homologous on $e_{1}$ with respect to $f$ and $\omega_{2}$.

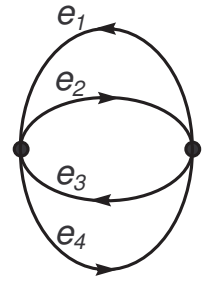

$G_{1}$

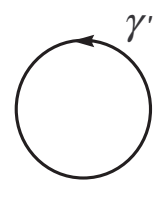

$G_{2}$

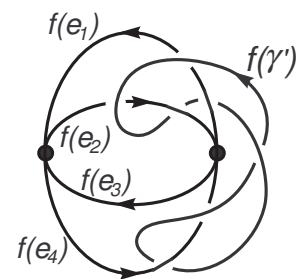

$f$

Figure 3.1. 
Example 3.2. Let $G=G_{1} \cup G_{2}$ be the graph as illustrated in Fig. 3.2. We denote the cycle of $G_{1}$ which contains $e_{i}$ and $e_{j}(1 \leq i, j \leq 4)$ by $\gamma_{i j}$. Let $\omega_{1}$ be the weight on $\Gamma\left(G_{1}\right)$ over $\mathbb{Z}$ defined by

$$
\omega_{1}(\gamma)= \begin{cases}1 & \left(\gamma=\gamma_{14}, \gamma_{23}\right) \\ -1 & \left(\gamma=\gamma_{13}, \gamma_{24}\right) \\ 0 & (\text { otherwise })\end{cases}
$$

and $\omega_{2}$ the weight on $\Gamma\left(G_{2}\right)$ over $\mathbb{Z}$ defined by $\omega_{2}\left(\gamma^{\prime}\right)=1$. Let $f$ be the spatial embedding of $G$ as illustrated in Fig. 3.2, Note that

$$
\Gamma_{e_{1}, e_{5}}\left(G_{1}\right)=\left\{\gamma_{13}, \gamma_{14}\right\}=\left\{e_{1}+e_{5}-e_{3}-e_{6}, e_{1}+e_{5}-e_{4}-e_{6}\right\}
$$

and

$$
\sum_{\gamma \in \Gamma_{e_{1}, e_{5}}\left(G_{1}\right)} \omega_{1}(\gamma) \gamma=-\left(e_{1}+e_{5}-e_{3}-e_{6}\right)+\left(e_{1}+e_{5}-e_{4}-e_{6}\right)=e_{3}-e_{4} .
$$

Then we have that

$$
\operatorname{lk}\left(\sum_{\gamma \in \Gamma_{e_{1}, e_{5}}\left(G_{1}\right)} \omega_{1}(\gamma) f(\gamma), f\left(\gamma^{\prime}\right)\right)=\operatorname{lk}\left(f\left(e_{3}-e_{4}\right), f\left(\gamma^{\prime}\right)\right)=0 .
$$

Therefore $\omega_{1}$ is null-homologous on a pair of adjacent edges $e_{1}$ and $e_{5}$ with respect to $f$ and $\omega_{2}$.

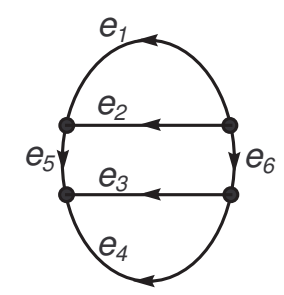

$G_{1}$

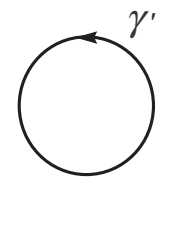

$G_{2}$

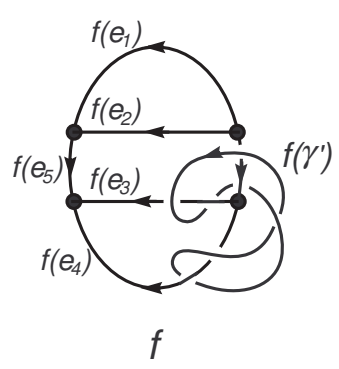

FiguRE 3.2 .

Now let $G=G_{1} \cup G_{2}$ be a disjoint union of two graphs, $\omega_{i}$ a weight on $\Gamma\left(G_{i}\right)$ over $\mathbb{Z}_{n}(i=1,2)$ and $f$ a spatial embedding of $G$. For $\gamma \in \Gamma\left(G_{1}\right)$ and $\gamma^{\prime} \in \Gamma\left(G_{2}\right)$, we put

$$
\eta\left(f(\gamma), f\left(\gamma^{\prime}\right)\right)=\frac{1}{6}\left(m^{3}-m\right)
$$

where $m=\operatorname{lk}\left(f(\gamma), f\left(\gamma^{\prime}\right)\right)$ under arbitrary orientations of $\gamma$ and $\gamma^{\prime}$. Then we define that

$\tilde{\eta}_{\omega_{1}, \omega_{2}}(f)=\operatorname{gcd}\left\{\eta\left(f(\gamma), f\left(\gamma^{\prime}\right)\right) \mid \gamma \in \Gamma\left(G_{1}\right), \gamma^{\prime} \in \Gamma\left(G_{2}\right), \omega_{1}(\gamma) \omega_{2}\left(\gamma^{\prime}\right) \not \equiv 0 \quad(\bmod n)\right\}$, where gcd means the greatest common divisor. Note that $\tilde{\eta}_{\omega_{1}, \omega_{2}}(f)$ is a well-defined non-negative integer which does not depends on the choice of orientations of each 
pair of disjoint cycles. Then we define $\tilde{\beta}_{\omega_{1}, \omega_{2}}(f) \in \mathbb{Z}_{n}$ by

$$
\tilde{\beta}_{\omega_{1}, \omega_{2}}(f) \equiv \sum_{\substack{\gamma \in \Gamma\left(G_{1}\right) \\ \gamma^{\prime} \in \Gamma\left(G_{2}\right)}} \omega_{1}(\gamma) \omega_{2}\left(\gamma^{\prime}\right) \tilde{\beta}\left(f(\gamma), f\left(\gamma^{\prime}\right)\right) \quad\left(\bmod \operatorname{gcd}\left\{n, \tilde{\eta}_{\omega_{1}, \omega_{2}}(f)\right\}\right)
$$

Here we may calculate $\tilde{\beta}\left(f(\gamma), f\left(\gamma^{\prime}\right)\right)$ under arbitrary orientations of $\gamma$ and $\gamma^{\prime}$.

Remark 3.3. (1) For an oriented 2-component link $L, \tilde{\beta}(L)$ is not a link-homotopy invariant of $L$. Thus $\tilde{\beta}_{\omega_{1}, \omega_{2}}(f)$ may be not an edge (resp. vertex)-homotopy invariant of $f$ as it is. See also Remark 4.6.

(2) By Theorem 2.2, the value of $\tilde{\beta}\left(f(\gamma), f\left(\gamma^{\prime}\right)\right)$ is well-defined modulo $\eta\left(f(\gamma), f\left(\gamma^{\prime}\right)\right)$. This is the reason why we consider the modulo $\tilde{\eta}_{\omega_{1}, \omega_{2}}(f)$ reduction.

Then, let us state the invariance of $\tilde{\beta}_{\omega_{1}, \omega_{2}}$ up to edge (resp. vertex)-homotopy under some conditions on graphs and its spatial embeddings.

Theorem 3.4. (1) If $\omega_{i}$ is weakly balanced on any edge of $G_{i}$ and null-homologous on any edge of $G_{i}$ with respect to $f$ and $\omega_{j}(i=1,2, i \neq j)$, then $\tilde{\beta}_{\omega_{1}, \omega_{2}}(f)$ is an edge-homotopy invariant of $f$.

(2) If $\omega_{i}$ is weakly balanced on any pair of adjacent edges of $G_{i}$ and null-homologous on any pair of adjacent edges of $G_{i}$ with respect to $f$ and $\omega_{j}(i=1,2, i \neq j)$, then $\tilde{\beta}_{\omega_{1}, \omega_{2}}(f)$ is a vertex-homotopy invariant of $f$.

Proof. (1) Let $f$ and $g$ be two spatial embeddings of $G$ such that $g$ is edge-homotopic to $f$. Then it holds that

$$
\tilde{\eta}_{\omega_{1}, \omega_{2}}(f)=\tilde{\eta}_{\omega_{1}, \omega_{2}}(g)
$$

because the linking number of a constituent 2-component link of a spatial graph is an edge-homotopy invariant. First we show that if $f$ is transformed into $g$ by self crossing changes on $f\left(G_{1}\right)$ and ambient isotopies then $\tilde{\beta}_{\omega_{1}, \omega_{2}}(f)=\tilde{\beta}_{\omega_{1}, \omega_{2}}(g)$. It is clear that any link invariant of a constituent link of a spatial graph is also an ambient isotopy invariant of the spatial graph. Thus we may assume that $g$ is obtained from $f$ by a single crossing change on $f(e)$ for an edge $e$ of $G_{1}$ as illustrated in Fig. 3.3. Moreover, by smoothing on this crossing point we can obtain the spatial embedding $h$ of $G$ and the knot $J_{h}$ as illustrated in Fig. 3.3. Then by (3.1), Lemma 
2.1 and the assumptions for $\omega_{1}$, we have that

$$
\begin{aligned}
& =\sum_{\substack{\gamma \in \Gamma_{e}\left(G_{1}\right) \\
\gamma^{\prime} \in \Gamma\left(G_{2}\right)}} \omega_{1}(\gamma) \omega_{2}\left(\gamma^{\prime}\right)\left\{\tilde{\beta}\left(f(\gamma), f\left(\gamma^{\prime}\right)\right)-\tilde{\beta}\left(g(\gamma), g\left(\gamma^{\prime}\right)\right)\right\} \\
= & \sum_{\substack{\gamma \in \Gamma_{e}\left(G_{1}\right) \\
\gamma^{\prime} \in \Gamma\left(G_{2}\right)}} \omega_{1}(\gamma) \omega_{2}\left(\gamma^{\prime}\right) \operatorname{lk}\left(h\left(\gamma^{\prime}\right), J_{h}\right)\left\{\operatorname{lk}\left(f(\gamma), f\left(\gamma^{\prime}\right)\right)-\operatorname{lk}\left(h\left(\gamma^{\prime}\right), J_{h}\right)\right\} \\
= & \sum_{\gamma^{\prime} \in \Gamma\left(G_{2}\right)} \omega_{2}\left(\gamma^{\prime}\right)\left\{\operatorname{lk}\left(h\left(\gamma^{\prime}\right), J_{h}\right) \sum_{\gamma \in \Gamma_{e}\left(G_{1}\right)} \omega_{1}(\gamma) \operatorname{lk}\left(f(\gamma), f\left(\gamma^{\prime}\right)\right)\right. \\
= & \left.-\sum_{\gamma \in \Gamma_{e}\left(G_{1}\right)} \omega_{1}(\gamma) \operatorname{lk}\left(h\left(\gamma^{\prime}\right), J_{h}\right)^{2}\right\} \\
& \left.\sum_{\gamma^{\prime} \in \Gamma\left(G_{2}\right)} \omega_{2}\left(\gamma^{\prime}\right)\left\{\operatorname{lk}\left(h\left(\gamma^{\prime}\right), J_{h}\right) \operatorname{lk}\right\} \sum_{\gamma \in \Gamma_{e}\left(G_{1}\right)} \omega_{1}(\gamma) f(\gamma), f\left(\gamma^{\prime}\right)\right) \\
& \left.-\operatorname{lk}\left(h\left(\gamma^{\prime}\right), J_{h}\right)^{2}\left(\sum_{\gamma \in \Gamma_{e}\left(G_{1}\right)} \omega_{1}(\gamma)\right)\right\} \\
\equiv & 0 \quad\left(\bmod \operatorname{gcd}\left\{n, \tilde{\eta}_{\omega_{1}, \omega_{2}}(f)\right\}\right) .
\end{aligned}
$$

Therefore we have that $\tilde{\beta}_{\omega_{1}, \omega_{2}}(f)=\tilde{\beta}_{\omega_{1}, \omega_{2}}(g)$. In the same way we can show that if $f$ is transformed into $g$ by self crossing changes on $f\left(G_{2}\right)$ and ambient isotopies then $\tilde{\beta}_{\omega_{1}, \omega_{2}}(f)=\tilde{\beta}_{\omega_{1}, \omega_{2}}(g)$. Thus we have that $\tilde{\beta}_{\omega_{1}, \omega_{2}}(f)$ is an edge-homotopy invariant of $f$.
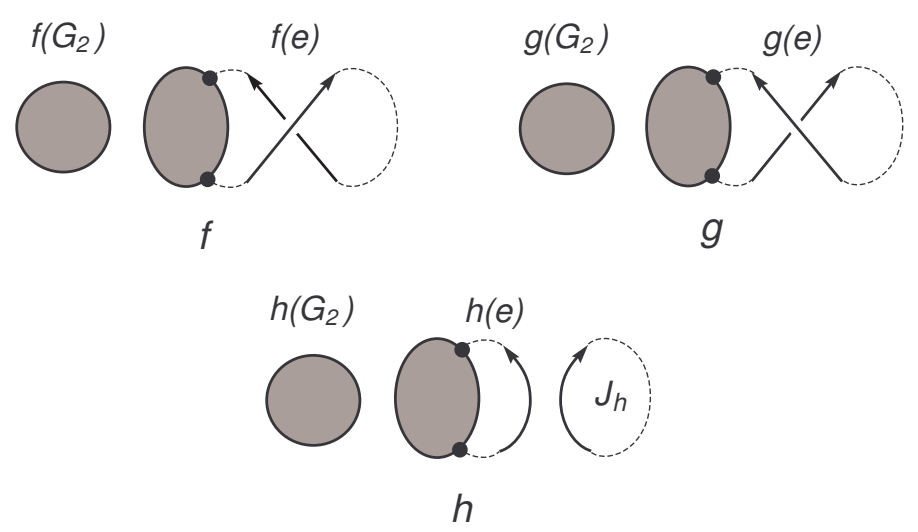

FIGURE 3.3.

(2) By considering the triple of spatial embeddings as illustrated in Fig. 3.4, we can prove (2) in a similar way as the proof of (1). We omit the details. 


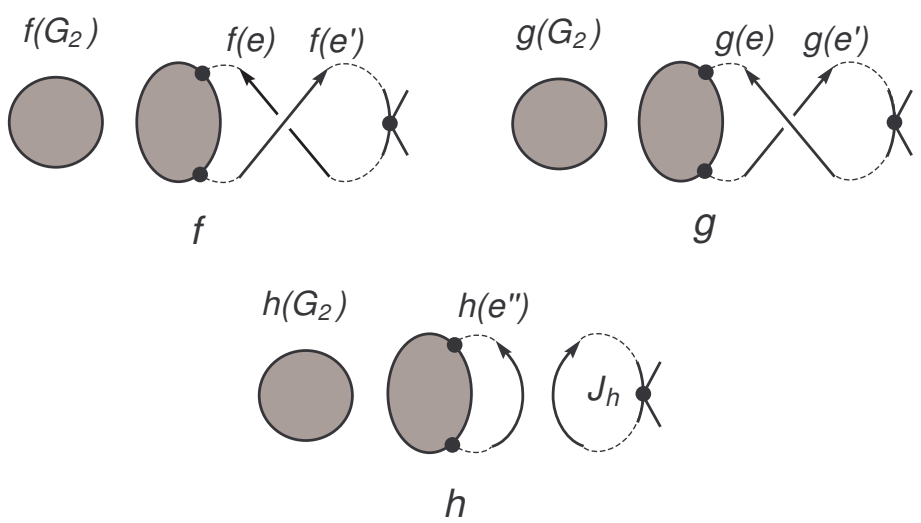

FIGURE 3.4 .

Remark 3.5. In particular, if it holds that

$$
\omega_{1}(\gamma) \omega_{2}\left(\gamma^{\prime}\right) \operatorname{lk}\left(f(\gamma), f\left(\gamma^{\prime}\right)\right)=0
$$

for any $\gamma \in \Gamma\left(G_{1}\right)$ and $\gamma^{\prime} \in \Gamma\left(G_{2}\right)$, then $\tilde{\beta}_{\omega_{1}, \omega_{2}}(f)$ coincides with Fleming and the author's invariant $\beta_{\omega_{1}, \omega_{2}}(f)$ defined in [4].

\section{EXAmples}

Let $G$ be a planar graph which is not a cycle. An embedding $p: G \rightarrow \mathbb{S}^{2}$ is said to be cellular if the closure of each of the connected components of $\mathbb{S}^{2}-p(G)$ on $\mathbb{S}^{2}$ is homeomorphic to the disk. Then we regard the set of the boundaries of all of the connected components of $\mathbb{S}^{2}-p(G)$ as a subset of $\Gamma(G)$ and denote it by $\Gamma_{p}(G)$. We say that $G$ admits a checkerboard coloring on $\mathbb{S}^{2}$ if there exists a cellular embedding $p: G \rightarrow \mathbb{S}^{2}$ such that we can color all of the connected components of $\mathbb{S}^{2}-p(G)$ by two colors (black and white) so that any of the two components which are adjacent by at least one edge have distinct colors. We denote the subset of $\Gamma_{p}(G)$ which corresponds to the black (resp. white) colored components by $\Gamma_{p}^{b}(G)$ (resp. $\left.\Gamma_{p}^{w}(G)\right)$. Then, for any edge $e$ of $G$, there exist exactly two cycles $\gamma \in \Gamma_{p}^{b}(G)$ and $\gamma^{\prime} \in \Gamma_{p}^{w}(G)$ such that $e \subset \gamma$ and $e \subset \gamma^{\prime}$. Thus we have the following immediately.

Proposition 4.1. Let $G$ be a planar graph which is not a cycle and admits a checkerboard coloring on $\mathbb{S}^{2}$ with respect to a cellular embedding $p: G \rightarrow \mathbb{S}^{2}$. Let $\omega_{p}$ be the weight on $\Gamma(G)$ over $\mathbb{Z}_{n}$ defined by

$$
\omega_{p}(\gamma)= \begin{cases}1 & \left(\gamma \in \Gamma_{p}^{b}(G)\right) \\ n-1 & \left(\gamma \in \Gamma_{p}^{w}(G)\right) \\ 0 & \left(\gamma \in \Gamma(G)-\Gamma_{p}(G)\right) .\end{cases}
$$

Then $\omega_{p}$ is weakly balanced on any edge of $G$.

We call the weight $\omega_{p}$ in Proposition 4.1 a checkerboard weight. Moreover, by giving the counter clockwise orientation to each $p(\gamma)$ for $\gamma \in \Gamma_{p}^{b}(G)$ and the clockwise orientation to each $p(\gamma)$ for $\gamma \in \Gamma_{p}^{w}(G)$ with respect to the orientation of $\mathbb{S}^{2}$, an orientation is given for each edge of $G$ naturally. We call this orientation of $G$ a checkerboard orientation over the checkerboard coloring. Since the orientation of 
each edge $e$ is coherent with the orientation of each cycle $\gamma \in \Gamma_{p}(G)$ which contains $e$, by Theorem 3.4 we have the following.

Theorem 4.2. Let $G=G_{1} \cup G_{2}$ be a disjoint union of two planar graphs such that $G_{i}$ is not a cycle and admits a checkerboard coloring on $\mathbb{S}^{2}$ with respect to a cellular embedding $p_{i}: G \rightarrow \mathbb{S}^{2}(i=1,2)$. Let $\omega_{p_{i}}$ be the checkerboard weight on $\Gamma\left(G_{i}\right)$ over $\mathbb{Z}_{n}(i=1,2)$. We orient $G$ by the checkerboard orientation of $G_{i}$ over the checkerboard coloring $(i=1,2)$. Then, for a spatial embedding $f$ of $G$, if $\omega_{i}$ is null-homologous on any edge of $G_{i}$ with respect to $f$ and $\omega_{j}(i=1,2, i \neq j)$, then $\tilde{\beta}_{\omega_{1}, \omega_{2}}(f)(\bmod n)$ is an edge-homotopy invariant of $f$.

Example 4.3. Let $G=G_{1} \cup G_{2}$ be a disjoint union of two planar graphs as in Theorem 4.2 and $f$ a spatial embedding of $G$. Let $\omega_{p_{i}}: \Gamma\left(G_{i}\right) \rightarrow \mathbb{Z}_{n}$ be the checkerboard weight $(i=1,2)$, where

$$
n=\operatorname{gcd}\left\{\operatorname{lk}\left(f(\gamma), f\left(\gamma^{\prime}\right)\right) \mid \gamma \in \Gamma_{p_{1}}\left(G_{1}\right), \gamma^{\prime} \in \Gamma_{p_{2}}\left(G_{2}\right)\right\} .
$$

Then, for any edge $e$ of $G_{i}$ and any $\gamma^{\prime} \in \Gamma_{p_{j}}\left(G_{j}\right)(i \neq j)$, we have that

$$
\mathrm{lk}\left(\sum_{\gamma \in \Gamma_{e}\left(G_{i}\right)} \omega_{i}(\gamma) f(\gamma), f\left(\gamma^{\prime}\right)\right)=\sum_{\gamma \in \Gamma_{e}\left(G_{i}\right)} \omega_{i}(\gamma) \operatorname{lk}\left(f(\gamma), f\left(\gamma^{\prime}\right)\right) \equiv 0 \quad(\bmod n) .
$$

Thus we have that $\omega_{i}$ is null-homologous on any edge of $G_{i}$ with respect to $f$ and $\omega_{j}$ $(i=1,2, i \neq j)$. Therefore we have that $\tilde{\beta}_{\omega_{p_{1}}, \omega_{p_{2}}}(f)(\bmod n)$ is an edge-homotopy invariant of $f$.

For example, let $\Theta_{4}$ be the graph with two vertices $u$ and $v$ and 4 edges $e_{1}, e_{2}, e_{3}, e_{4}$ each of which joins $u$ and $v$. We denote the cycle of $\Theta_{4}$ consists of two edges $e_{i}$ and $e_{j}$ by $\gamma_{i j}$. Let $p: \Theta_{4} \rightarrow \mathbb{S}^{2}$ be the cellular embedding as illustrated in the left-hand side of Fig. 4.1. It is clear that $\Theta_{4}$ admits the checkerboard coloring on $\mathbb{S}^{2}$ with respect to $p$ as illustrated in the center of Fig. 4.1. The right-hand side of Fig. 4.1 shows the checkerboard orientation of $\Theta_{4}$ over the checkerboard coloring.

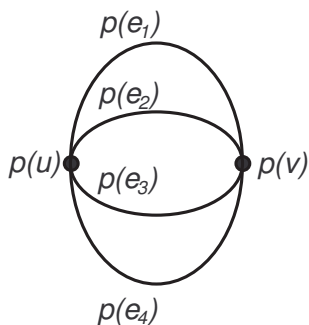

$p$

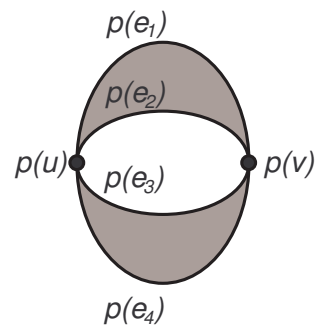

checkerboard
coloring

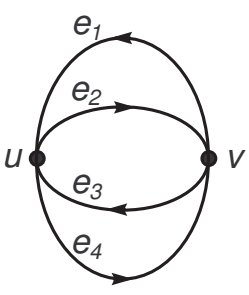

checkerboard orientation

FIGURE 4.1 .

Let $G=\Theta_{4}^{1} \cup \Theta_{4}^{2}$ be a disjoint union of two copies of $\Theta_{4}$. For a non-negative integer $m$, let $f_{m}$ and $g_{m}$ be two spatial embeddings of $G$ as illustrated in Fig. 4.2 Note that

$$
\operatorname{lk}\left(f_{m}(\gamma), f_{m}\left(\gamma^{\prime}\right)\right)=\operatorname{lk}\left(g_{m}(\gamma), g_{m}\left(\gamma^{\prime}\right)\right)=0 \text { or } m
$$


for any $\gamma \in \Gamma\left(\Theta_{4}^{1}\right)$ and $\gamma^{\prime} \in \Gamma\left(\Theta_{4}^{2}\right)$. So we have that $n=m$. Let $\omega_{i}: \Gamma\left(\Theta_{4}^{i}\right) \rightarrow \mathbb{Z}_{m}$ be the checkerboard weight $(i=1,2)$. Then, by a direct calculation we can see that the constituent 2-component link of $f_{m}$ which has a non-zero generalized Sato-Levine invariant is only $L=f_{m}\left(\gamma_{14} \cup \gamma_{14}^{\prime}\right)$ and $\tilde{\beta}(L)=2$. Actually the other constituent 2component link $f_{m}\left(\gamma \cup \gamma^{\prime}\right)$ for $\gamma \in \Gamma_{p}\left(\Theta_{4}^{1}\right)$ and $\gamma^{\prime} \in \Gamma_{p}\left(\Theta_{4}^{2}\right)$ is a trivial 2-component link or $T_{m}^{\prime}$ as illustrated in Fig. 2.2. Thus we have that $\tilde{\beta}_{\omega_{1}, \omega_{2}}\left(f_{m}\right) \equiv 2(\bmod m)$. On the other hand, we can see that each constituent 2-component link $g_{m}\left(\gamma \cup \gamma^{\prime}\right)$ for $\gamma \in \Gamma_{p}\left(\Theta_{4}^{1}\right)$ and $\gamma^{\prime} \in \Gamma_{p}\left(\Theta_{4}^{2}\right)$ is a trivial 2-component link or $T_{m}^{\prime}$. Thus we have that $\tilde{\beta}_{\omega_{1}, \omega_{2}}\left(g_{m}\right) \equiv 0(\bmod m)$. Therefore we have that $f_{m}$ and $g_{m}$ are not edge-homotopic if $m \neq 1,2$. We remark here that the case of $m=0$ has already shown by Fleming and the author in [4, Example 4.3].
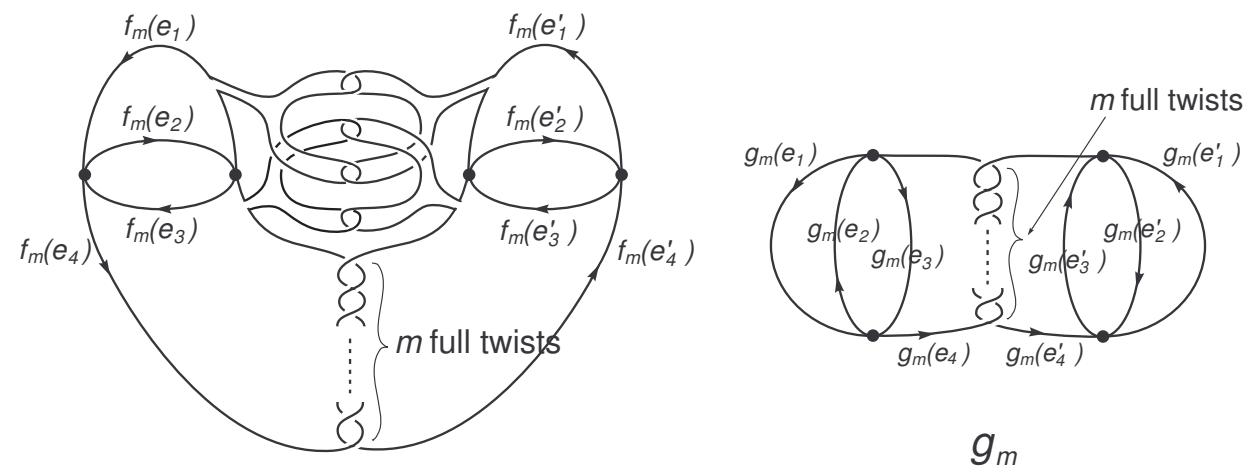

$f_{m}$

$g_{m}$

FiguRe 4.2 .

Example 4.4. Let $G=\Theta_{4}^{1} \cup \Theta_{4}^{2}$ be a disjoint union of two copies of $\Theta_{4}$ oriented in the same way as Example 4.3 and $\omega_{i}: \Gamma\left(\Theta_{4}^{i}\right) \rightarrow \mathbb{Z}$ the checkerboard weight $(i=1,2)$. For $\Theta_{4}^{1}$, we have that

$$
\begin{gathered}
\sum_{\gamma \in \Gamma_{e_{1}}\left(\Theta_{4}^{1}\right)} \omega_{1}(\gamma) \gamma=e_{2}-e_{4}, \quad \sum_{\gamma \in \Gamma_{e_{2}}\left(\Theta_{4}^{1}\right)} \omega_{1}(\gamma) \gamma=e_{1}-e_{3}, \\
\sum_{\gamma \in \Gamma_{e_{3}}\left(\Theta_{4}^{1}\right)} \omega_{1}(\gamma) \gamma=e_{4}-e_{2}, \quad \sum_{\gamma \in \Gamma_{e_{4}}\left(\Theta_{4}^{1}\right)} \omega_{1}(\gamma) \gamma=e_{3}-e_{1} .
\end{gathered}
$$

This implies that $\omega_{1}$ is null-homologous on any edge of $G_{1}$ with respect to a spatial embedding $f$ of $G$ and $\omega_{2}$ if and only if

$$
\left.\left.\mathrm{lk}\left(f\left(\gamma_{13}\right), f\left(\gamma^{\prime}\right)\right)\right)=\mathrm{lk}\left(f\left(\gamma_{24}\right), f\left(\gamma^{\prime}\right)\right)\right)=0
$$

for any $\gamma^{\prime} \in \Gamma_{p}\left(\Theta_{4}^{2}\right)$. The same condition can be said of $\omega_{2}$. For an integer $m$, let $f_{m}$ be the spatial embedding of $G$ as illustrated in Fig. 4.3, Note that

$$
\operatorname{lk}\left(f_{k}(\gamma), f_{k}\left(\gamma^{\prime}\right)\right)=\operatorname{lk}\left(f_{l}(\gamma), f_{l}\left(\gamma^{\prime}\right)\right)=0 \text { or } 1(k \neq l)
$$

for any $\gamma \in \Gamma\left(\Theta_{4}^{1}\right)$ and $\gamma^{\prime} \in \Gamma\left(\Theta_{4}^{2}\right)$. Since we can see that $\omega_{i}$ satisfies (4.1), we have that $\omega_{i}$ is null-homologous on any edge of $G_{i}$ with respect to $f_{m}$ and $\omega_{j}(i=$ $1,2, i \neq j)$. Namely $\tilde{\beta}_{\omega_{1}, \omega_{2}}\left(f_{m}\right)$ is an integer-valued edge-homotopy invariant of $f_{m}$. 
Then, by a direct calculation we can see that the constituent 2-component link of $f_{m}$ which has a non-zero generalized Sato-Levine invariant is only $L=f_{m}\left(\gamma_{14} \cup \gamma_{14}^{\prime}\right)$ and $\tilde{\beta}(L)=2 m$. Actually the other constituent 2-component link $f_{m}\left(\gamma \cup \gamma^{\prime}\right)$ for $\gamma \in \Gamma_{p}\left(\Theta_{4}^{1}\right)$ and $\gamma^{\prime} \in \Gamma_{p}\left(\Theta_{4}^{2}\right)$ is a Hopf link. Thus we have that $\tilde{\beta}_{\omega_{1}, \omega_{2}}\left(f_{m}\right)=2 m$. Therefore we have that $f_{k}$ and $f_{l}$ are not edge-homotopic for $k \neq l$.

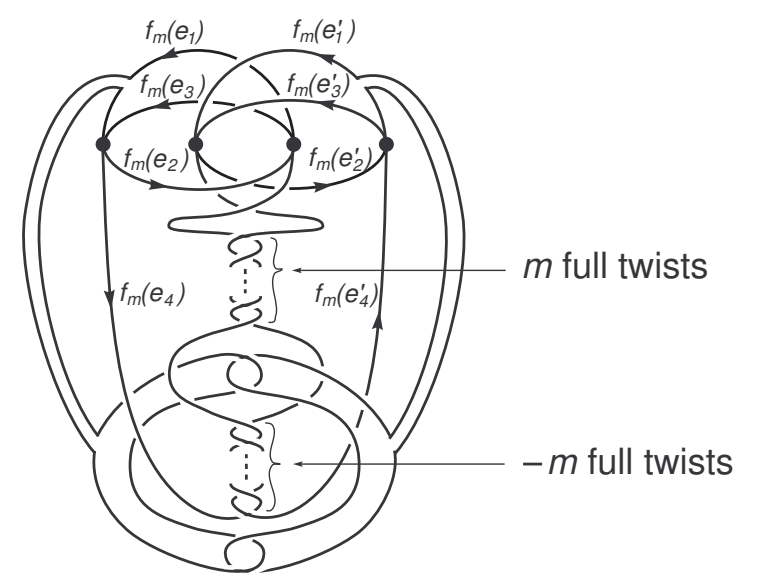

FigURE 4.3 .

Example 4.5. Let $H$ be the same oriented graph as $G_{1}$ in Fig. 3.2. We denote the cycle of $H$ which contains $e_{i}$ and $e_{j}$ by $\gamma_{i j}$. Let $G=H^{1} \cup H^{2}$ be a disjoint union of two copies of $H$ and $f_{m}$ the spatial embedding of $G$ as illustrated in Fig. 4.4. This spatial embedding $f_{m}$ contains exactly one constituent 4-component link $L_{m}=f_{m}\left(\gamma_{12} \cup \gamma_{34} \cup \gamma_{12}^{\prime} \cup \gamma_{34}^{\prime}\right)$. By calculating Milnor's $\mu$-invariant 12] of length 4 of $L_{m}$, it can be shown that $f_{k}$ and $f_{l}$ are not vertex-homotopic for $k \neq l$. But we can also prove this fact by our vertex-homotopy invariant as follows. Let $\omega$ be the same weight on $\Gamma(H)$ over $\mathbb{Z}$ as $\omega_{1}$ in Example 3.2 We define the weight $\omega_{i}$ of $\Gamma\left(H^{i}\right)$ over $\mathbb{Z}$ in the same way as $\omega(i=1,2)$. It is easy to see that $\omega_{i}$ is weakly balanced on any pair of adjacent edges of $H^{i}(i=1,2)$. Moreover, we have that

$$
\begin{aligned}
& \sum_{\gamma \in \Gamma_{e_{1}, e_{5}}(H)} \omega(\gamma) \gamma=e_{3}-e_{4}, \sum_{\gamma \in \Gamma_{e_{1}, e_{2}}(H)} \omega(\gamma) \gamma=0, \\
& \sum_{\gamma \in \Gamma_{e_{1}, e_{6}}(H)} \omega(\gamma) \gamma=e_{3}-e_{4}, \sum_{\gamma \in \Gamma_{e_{2}, e_{5}}(H)} \omega(\gamma) \gamma=e_{4}-e_{3}, \\
& \sum_{\gamma \in \Gamma_{e_{2}, e_{6}}(H)} \omega(\gamma) \gamma=e_{4}-e_{3}, \sum_{\gamma \in \Gamma_{e_{3}, e_{5}}(H)} \omega(\gamma) \gamma=e_{1}-e_{2}, \\
& \sum_{\gamma \in \Gamma_{e_{3}, e_{6}}(H)} \omega(\gamma) \gamma=e_{1}-e_{2}, \quad \sum_{\gamma \in \Gamma_{e_{3}, e_{4}}(H)} \omega(\gamma) \gamma=0, \\
& \sum_{\gamma \in \Gamma_{e_{4}, e_{5}}(H)} \omega(\gamma) \gamma=e_{2}-e_{1}, \sum_{\gamma \in \Gamma_{e_{4}, e_{6}}(H)} \omega(\gamma) \gamma=e_{2}-e_{1} .
\end{aligned}
$$


This implies that $\omega_{1}$ is null-homologous on any pair of adjacent edges of $H^{1}$ with respect to a spatial embedding $f$ of $G$ and $\omega_{2}$ if and only if

$$
\left.\left.\operatorname{lk}\left(f\left(\gamma_{12}\right), f\left(\gamma^{\prime}\right)\right)\right)=\operatorname{lk}\left(f\left(\gamma_{34}\right), f\left(\gamma^{\prime}\right)\right)\right)=0
$$

for any $\gamma^{\prime} \in \Gamma\left(H^{2}\right)$ and $\omega_{2}\left(\gamma^{\prime}\right) \neq 0$. The same condition can be said of $\omega_{2}$. Then we can see that $\omega_{i}$ satisfies (4.2) for $f_{m}$, namely $\omega_{i}$ is null-homologous on any edge of $G_{i}$ with respect to $f_{m}$ and $\omega_{j}(i=1,2, i \neq j)$. Namely $\tilde{\beta}_{\omega_{1}, \omega_{2}}\left(f_{m}\right)$ is a vertex-homotopy invariant of $f_{m}$. Since the linking number of each constituent 2component link of $f_{m}$ is 0 or \pm 1 , we have that $\tilde{\eta}_{\omega_{1}, \omega_{2}}\left(f_{m}\right)=0$. Namely $\tilde{\beta}_{\omega_{1}, \omega_{2}}\left(f_{m}\right)$ is integer-valued. By a direct calculation we have that $\tilde{\beta}_{\omega_{1}, \omega_{2}}\left(f_{m}\right)=2 m$ in the same way as Example 4.4. Therefore we have that $f_{k}$ and $f_{l}$ are not vertex-homotopic for $k \neq l$.

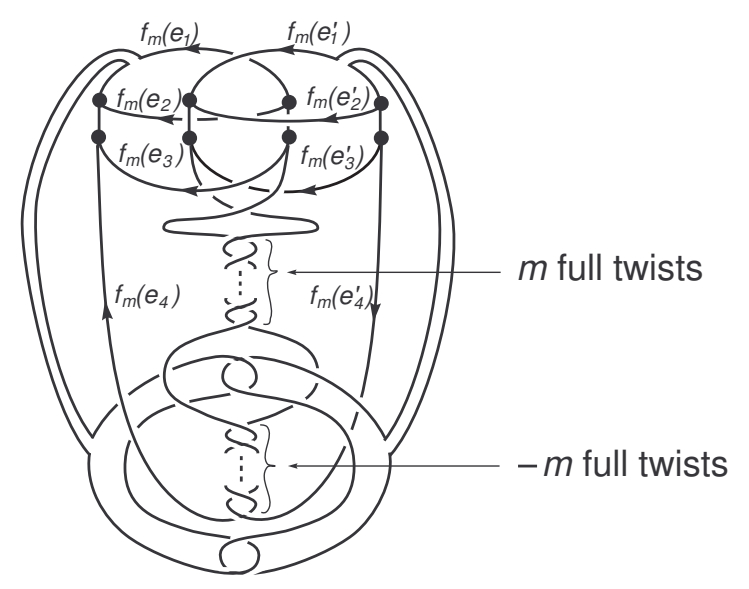

Figure 4.4 .

Remark 4.6. In Theorems 3.4 and 4.2 , the condition " $\omega_{i}$ is null-homologous on any edge of $G_{i}$ with respect to $f$ and $\omega_{j}(i=1,2, i \neq j)$ " is essential. Let $G=\Theta_{4}^{1} \cup \Theta_{4}^{2}$ be a disjoint union of two copies of $\Theta_{4}$ oriented in the same way as Example 4.4 and $\omega_{i}: \Gamma\left(\Theta_{4}^{i}\right) \rightarrow \mathbb{Z}$ the checkerboard weight $(i=1,2)$. Let $f$ and $g$ be two spatial embeddings of $G$ as illustrated in Fig. 4.5. Note that $f$ and $g$ are edge-homotopic. But by a direct calculation we have that $\tilde{\beta}_{\omega_{1}, \omega_{2}}(f)=-1$ and $\tilde{\beta}_{\omega_{1}, \omega_{2}}(g)=0$, namely $\tilde{\beta}_{\omega_{1}, \omega_{2}}(f)$ is not an edge-homotopy invariant of $f$. Actually $\omega_{1}$ is not null-homologous on $e_{4}$ with respect to $f$ and $\omega_{2}$.

\section{ACKNOWLEDGMENT}

The author would like to thank Professor Kouki Taniyama for informing him about the results in [18. He is also grateful to Doctor Thomas Fleming for his valuable comments.

\section{REFERENCES}

[1] P. M. Akhmet'iev, On a higher analog of the linking number of two curves, Topics in quantum groups and finite-type invariants, 113-127, Amer. Math. Soc. Transl. Ser. 2, 185, Amer. Math. Soc., Providence, RI, 1998. 


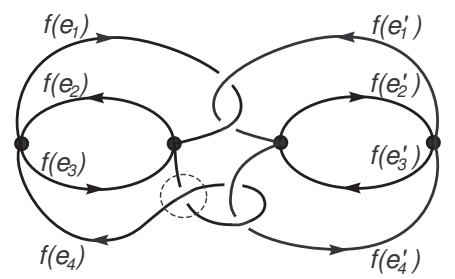

$f$

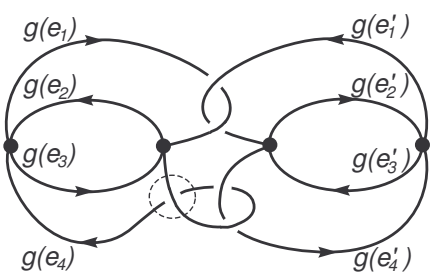

9

FIGURE 4.5 .

[2] P. M. Akhmet'iev and D. Repovš, A generalization of the Sato-Levine invariant (Russian), Tr. Mat. Inst. Steklova 221 (1998), 69-80; translation in Proc. Steklov Inst. Math. 221 (1998), 60-70.

[3] T. D. Cochran, Concordance invariance of coefficients of Conway's link polynomial, Invent. Math. 82 (1985), 527-541.

[4] T. Fleming and R. Nikkuni, Homotopy on spatial graphs and the Sato-Levine invariant, to appear in Transactions of the American Mathematical Society. arXiv:math.GT/0509003

[5] R. Hartley, The Conway potential function for links, Comment. Math. Helv. 58 (1983), 365378.

[6] F. Hosokawa, On $\nabla$-polynomials of links, Osaka Math. J. 10 (1958), 273-282.

[7] J. Hoste, The first coefficient of the Conway polynomial, Proc. Amer. Math. Soc. 95 (1985), 299-302.

[8] L. H. Kauffman, Formal knot theory, Mathematical Notes, 30, Princeton University Press, Princeton, NJ, 1983.

[9] T. Kanenobu, Y. Miyazawa and A. Tani, Vassiliev link invariants of order three, J. Knot Theory Ramifications 7 (1998), 433-462.

[10] P. Kirk and C. Livingston, Vassiliev invariants of two component links and the Casson-Walker invariant, Topology 36 (1997), 1333-1353.

[11] C. Livingston, Enhanced linking numbers, Amer. Math. Monthly 110 (2003), 361-385.

[12] J. Milnor, Link groups, Ann. of Math. (2) 59 (1954), 177-195.

[13] Y. Nakanishi, Delta link homotopy for two component links, Proceedings of the First Joint Japan-Mexico Meeting in Topology (Morelia, 1999), Topology Appl. 121 (2002), 169-182.

[14] N. Sato, Cobordisms of semiboundary links, Topology Appl. 18 (1984), 225-234.

[15] R. Sturm Beiss, The Arf and Sato link concordance invariants, Trans. Amer. Math. Soc. 322 (1990), 479-491.

[16] K. Taniyama, Link homotopy invariants of graphs in $R^{3}$, Rev. Mat. Univ. Complut. Madrid 7 (1994), 129-144.

[17] K. Taniyama, Cobordism, homotopy and homology of graphs in $R^{3}$, Topology 33 (1994), $509-523$.

[18] K. Taniyama, Graph-homotopy invariants, unpublished note.

Department of Mathematics, Faculty of Education, Kanazawa University, KakumaMaChi, KanazaWa, IshiKaWa, 920-1192, JaPan

E-mail address: nick@ed.kanazawa-u.ac.jp 